\title{
Evaluation of Acoustic Cavitation in Terephthalic Acid Solutions Containing Gold Nanoparticles by the Spectrofluorometry Method
}

\author{
Ameneh Sazgarnia ${ }^{1}$ and Ahmad Shanei ${ }^{2}$ \\ ${ }^{1}$ Research Center and Department of Medical Physics, School of Medicine, Mashhad University of Medical Sciences, \\ Vakil Abad boulevard, Mashhad 9177948564, Iran \\ ${ }^{2}$ Department of Medical Physics and Medical Engineering, School of Medicine, Isfahan University of Medical Sciences, \\ Darvaze Shiraz boulevard, Isfahan 8174673461, Iran
}

Correspondence should be addressed to Ahmad Shanei, shanei@med.mui.ac.ir

Received 29 April 2012; Revised 30 September 2012; Accepted 7 October 2012

Academic Editor: Korkut Yegin

Copyright ( $) 2012$ A. Sazgarnia and A. Shanei. This is an open access article distributed under the Creative Commons Attribution License, which permits unrestricted use, distribution, and reproduction in any medium, provided the original work is properly cited.

\begin{abstract}
Background. When a liquid is irradiated with high intensity and low-frequency ultrasound, acoustic cavitation occurs. The existence of particles in a liquid provides nucleation sites for cavitation bubbles and leads to a decrease in the ultrasonic intensity threshold needed for cavitation onset. Materials and Methods. The study was designed to measure hydroxyl radicals in terephthalic acid solutions containing gold nanoparticles in a near field of a $1 \mathrm{MHz}$ sonotherapy probe. The effect of ultrasound irradiation parameters containing mode of sonication and ultrasound intensity in hydroxyl radicals production have been investigated by the spectrofluorometry method. Results. Recorded fluorescence signal in terephthalic acid solution containing gold nanoparticles was higher than the terephthalic acid solution without gold nanoparticles. Also, the results showed that any increase in intensity of the sonication would be associated with an increase in the fluorescence intensity. Conclusion. Acoustic cavitation in the presence of gold nanoparticles has been introduced as a way for improving therapeutic effects on the tumors in sonodynamic therapy. Also, the terephthalic acid dosimetry is suitable for detecting and quantifying free hydroxyl radicals as a criterion of cavitation production over a certain range of conditions in medical ultrasound fields.
\end{abstract}

\section{Introduction}

In recent years the use of low-intensity ultrasound in therapeutic applications has become a developing field. One of the main fields of study is sonodynamic therapy (SDT) [1, $2]$. This new method of therapy has great potential because of its relatively easy application [1]. Although the mechanism of low-intensity ultrasound in treating malignant tissues is not well understood, the nonthermal effects of ultrasound, especially cavitation, is considered to be a primary reason for these purposes [3]. Cavitation can occur at stable and transient modes. In stable mode, the bubbles oscillate around an equilibrium radius during a considerable number of acoustic cycles without collapsing. In transient cavitation, bubbles grow rapidly and expand up to several times of their original size and violently collapse during a single acoustic compression cycle [4]. In fact, during the collapse, very high shear stresses and shock waves are produced. Moreover, very high pressure and temperature at the collapse region can produce free radicals, erosion, emulsification, molecular degradation, and sonoluminescence. This type of cavitation can be fatal tocells and is utilized to destroy cancer tumors [5].

On the basis of a few reports, the existence of a particle in a liquid provides a nucleation site for the cavitation bubble because of its surface roughness and leads to decrease in the cavitation threshold responsible for the increase in the quantity of bubbles, when the liquid is irradiated by ultrasound $[6,7]$. Thus, in this context, one approach is based on providing the nucleation sites that participate in 
the formation of cavities to reduce the threshold intensity required for cavitation.

Gold nanoparticles (GNPs) have been characterized as novel nanomaterials for use in cancer therapy because of their special optical properties $[8,9]$. Their low toxicity, good uptake by mammalian cells, and antiangiogenetic properties make GNPs highly attractive for medical applications [10].

In this study, the cavitation potential of GNPs has been studied via chemical dosimetry method [11] at therapeutic intensities of ultrasound.

Acoustic cavitation generates free radicals from the breakdown of water and other molecules. When water is sonicated, $\mathrm{OH}$ radicals are formed on thermolysis of $\mathrm{H}_{2} \mathrm{O}$. The initial step in the decomposition of water is the production of hydroxyl and hydrogen radicals. Simplified equations for production of free radicals by collapse of cavitation in water solutions are shown below [12]:

$$
\begin{gathered}
\mathrm{H}_{2} \mathrm{O} \longrightarrow \mathrm{H}^{\bullet}+{ }^{\bullet} \mathrm{OH} \\
\mathrm{H}_{2} \mathrm{O} \longrightarrow \mathrm{H}_{2}+{ }^{\bullet} \mathrm{O} \\
\mathrm{H}^{\bullet}+\mathrm{H}_{2} \mathrm{O} \longrightarrow \mathrm{H}_{2}+{ }^{\bullet} \mathrm{OH} \\
\mathrm{O}+\mathrm{H}_{2} \mathrm{O} \longrightarrow{ }^{\cdot} \mathrm{OH}+{ }^{\bullet} \mathrm{OH}
\end{gathered}
$$

Such chemical products also may be used to measure cavitation activity. It has been shown that terephthalic acid (TA) [benzene-1, 4-dicarboxylic acid] is suitable for detecting and quantifying free hydroxyl radicals generated by the collapse of cavitation bubbles in ultrasound irradiations. During this process, the TA solution as a dosimetric solution reacts with a hydroxyl radical generated through water sonolysis. Therefore, 2-hydroxyterephthalic acid (HTA) is produced which can be detected using fluorescence spectroscopy with excitation and emission wavelengths at 310 and $420 \mathrm{~nm}$, respectively [13].

\section{Materials and Methods}

2.1. Preparation and Characterization of Gold Nanoparticles. GNPs were synthesized according to standard wet chemical methods using sodium borohydride as a reducing agent [14]. In this experiment, $50 \mathrm{~mL}$ of aqueous solution containing $4.3 \mathrm{mg}$ of solid sodium borohydride was added to $100 \mathrm{~mL}$ of $100 \mu \mathrm{M}$ aqueous solution of tetracholoroauric acid under vigorous stirring which was continued overnight. GNPs thus formed were filtered through $0.22 \mu \mathrm{m}$ filter paper and used for the experiments [14]. TEM revealed that spherical GNPs of approximately $6-8 \mathrm{~nm}$ were formed by this method. Size distribution analysis showed that nearly $70 \%$ of the particles resided within 5 to $9 \mathrm{~nm}$ of size range.

2.2. Ultrasound Generator System. Ultrasound irradiation was conducted with a therapeutic ultrasound unit $(215 \mathrm{~A}$; coproduct of Novin Medical Engineering Co., Tehran, Iran; and EMS Co., Reading, Berkshire, England) in a continuous mode at a frequency of $1 \mathrm{MHz}$ with a maximum intensity of $2 \mathrm{~W} / \mathrm{cm}^{2}$ for 3 minutes. Acoustic calibration for the power of the device was performed in a degassed water tank, using an ultrasound balanced power meter (UPM 2000; Netech Corporation, Grand Rapids, MI) with uncertainty of $\pm 1 \mathrm{~mW}$. All quoted intensities were spatial averagetemporal average $\left(\mathrm{I}_{\text {SATA }}\right)$ in our experiments. An ultrasound transducer with a surface area of $7.0 \mathrm{~cm}^{2}$ was horizontally submerged in the bottom of a glass container filled with degassed water.

2.3. Preparation of Terephthalic Acid Solution. The dosimetry solution of TA was prepared according to the standard protocols, containing TA $(2 \mathrm{mmol} / \mathrm{L}$, Aldrich $)$ in almost $800 \mathrm{~mL}$ deionized water and then treated with $5 \mathrm{~mL} \mathrm{NaOH}$ (1 M). Ultrasonic irradiation experiments should be carried out in the presence of an alkaline buffer. To increase the $\mathrm{pH}$ value of the solution, $\mathrm{NaOH}$ was added to it. The solution was stirred for about one hour and kept in a cool and dark place $\left(4^{\circ} \mathrm{C}\right)$ to prevent photochemical reactions $[12,13]$. A cylindrical chamber was constructed from PVC in order to sonicate the TA solution $\left(5 \mathrm{~cm}^{3}\right)$. The chamber's floor was made from a thin acoustically transparent parafilm layer.

2.4. Experimental Protocol of Chemical Dosimetry. The chamber containing the TA solution was located in a container filled with degassed water in the near field of the probe at $5 \mathrm{~mm}$ distance from the surface of the probe. To perform experiments under progressive wave conditions and limit the action of acoustic reflection from the wall of the container, the inner surfaces of the anterior side of the probe was covered by absorbent ultrasound material [12]. The sonication time of the TA solution was selected at 20 min [12]. In such circumstances the maximum increase in temperature was $3^{\circ} \mathrm{C}\left(18-20^{\circ} \mathrm{C}\right)$. The measurements were performed on two TA solutions: TA solution containing GNPs and TA solution without GNPs. After ultrasound exposure at different intensities $\left(0.5,1\right.$ and $\left.2 \mathrm{~W} / \mathrm{cm}^{2}\right)$ in continuous and pulsed (in duty cycle of 50\%) modes on each solution, its fluorescence signal intensity was recorded using a spectrofluorimeter (FP-6200, Jasco, Japan) with excitation and emission wavelengths at 310 and $420 \mathrm{~nm}$, respectively. The irradiated solutions were kept in a dark place through the experiment and fluorimetric assessments were measured within 2 to 4 hours after sonication. Before ultrasound irradiation, the fluorescence signal of the solutions was also measured. Each experiment was repeated three times.

2.5. Data Analysis. A statistical analysis was performed using SPSS version 13.0 statistical software (SPSS Inc, Chicago, IL). According to the Kolmogorov-Smirnov normality test, the data distribution was normal. Consequently, the paired $t$-test was used to compare the fluorescence signal in two solutions with a confidence level of $95 \%$. The integrated fluorescence signal between different intensities was also compared using one way analysis of variance. Data are presented as Mean \pm SD. $P<0.05$ was considered as significant. 


\section{Results}

The fluorescence emission spectrum of TA solution without GNPs at $0.5,1$, and $2 \mathrm{~W} / \mathrm{cm}^{2}$ intensities in continuous mode is shown in Figure 1.

The fluorescence emission spectrum of TA solution containing GNPs at $0.5,1$, and $2 \mathrm{~W} / \mathrm{cm}^{2}$ intensities in continuous mode is shown in Figure 2.

The results of the fluorescence intensity in the TA solutions in the absence and presence of GNPs at $0.5,1$, and $2 \mathrm{~W} / \mathrm{cm}^{2}$ intensities in continuous mode are presented in Figure 3.

The fluorescence signal in the TA solution containing GNPs was significantly higher than the TA solution without GNPs in the different intensities in continuous mode $(P<$ $0.03)$.

There was a significant difference in the fluorescence signal intensity in the TA solution containing GNPs among all intensities $\left(0.5,1\right.$ and $\left.2 \mathrm{~W} / \mathrm{cm}^{2}\right)$ in continuous mode $(P<0.05)$, but there was no significant difference in the fluorescence intensity in TA solution without GNPs between 1 and $2 \mathrm{~W} / \mathrm{cm}^{2}$ intensities in continuous mode $(P>0.3)$.

The results of the fluorescence intensity in the TA solutions in the absence and presence of GNPs at $0.5,1$, and $2 \mathrm{~W} / \mathrm{cm}^{2}$ intensities in pulsed mode (in duty cycle of $50 \%$ ) are presented in Figure 4.

The fluorescence signal in the TA solution containing GNPs was significantly higher than the TA solution without GNPs at 1 and $2 \mathrm{~W} / \mathrm{cm}^{2}$ intensities in pulsed mode $(P<$ $0.05)$, but there was no significant difference in the fluorescence signal intensity between TA solution containing GNPs and TA solution without GNPs at $0.5 \mathrm{~W} / \mathrm{cm}^{2}$ intensity in pulsed mode $(P>0.06)$.

There was a significant difference in the fluorescence signal intensity in the TA solution containing GNPs among all intensities $\left(0.5,1\right.$ and $\left.2 \mathrm{~W} / \mathrm{cm}^{2}\right)$ in pulsed mode $(P<$ 0.04 ), but no significant difference in the fluorescence intensity in TA solution without GNPs between 1 and $2 \mathrm{~W} / \mathrm{cm}^{2}$ intensities in pulsed mode was observed $(P>0.4)$.

Experimental results related to the sonication mode of the TA solutions in the absence and presence of GNPs are shown in Figures 5 and 6.

Data indicated that any increase in intensity of the sonication will be associated with an increase in the fluorescence signal. Also, the recorded fluorescence signal in continuous mode of sonication was higher than the pulsed mode (in duty cycle of 50\%) at the same intensity. As seen, the level of fluorescence signal obtained using sonication of the TA solution in the absence and presence of GNPs at $2 \mathrm{~W} / \mathrm{cm}^{2}$ intensity in continuous mode is significantly higher than the pulsed mode $(P<0.05)$.

\section{Discussion}

There is considerable interest in the evaluation of cavitation for possibility of SDT which uses low-level ultrasound to release or enhance the action of therapeutic agents in vivo [1]. In order to quantify cavitation, certain methods are necessary, which are widely applicable methods. Existing

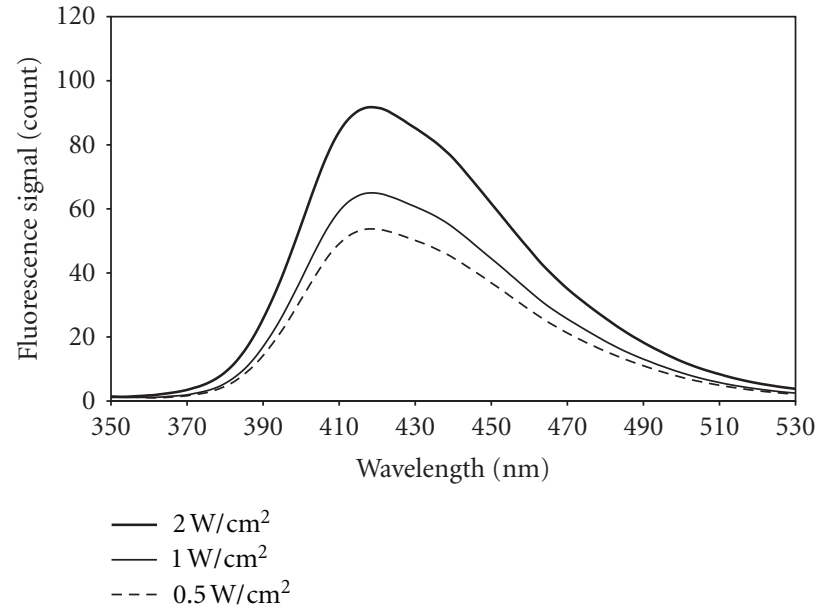

FIGURE 1: Recorded fluorescence emission spectrum from the TA solution without GNPs in the field of $1 \mathrm{MHz}$ ultrasound waves at intensities of $0.5,1$, and $2 \mathrm{~W} / \mathrm{cm}^{2}$ in continuous mode, excitation wavelength $=310 \mathrm{~nm}$, and emission and excitation band width $=$ $5 \mathrm{~nm}$.

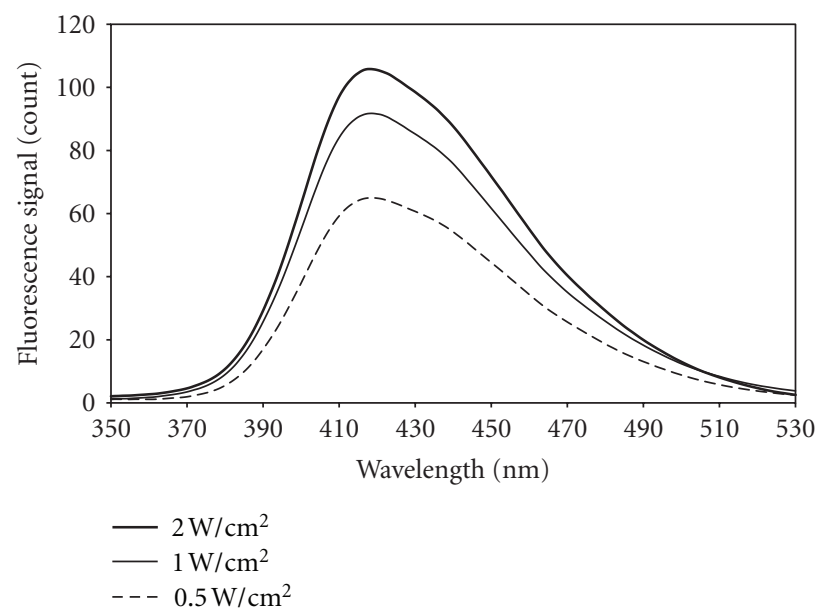

Figure 2: Recorded fluorescence emission spectrum from the TA solution containing GNPs in the field of $1 \mathrm{MHz}$ ultrasound waves at intensities of $0.5,1$, and $2 \mathrm{~W} / \mathrm{cm}^{2}$ in continuous mode, excitation wavelength $=310 \mathrm{~nm}$, and emission and excitation band width $=$ $5 \mathrm{~nm}$.

methods for performing these experiments include acoustic imaging and sonoluminescence whereas other methods such as the electron spin resonance (ESR) and laser holography are difficult and expensive [15]. The ESR is an extremely sensitive method for detecting the radicals produced but its application needs specialist and expensive equipment [15]. Other methods for monitoring the chemical effects of cavitation and free radicals produced are suitable to detect radical species [16]. Currently, cancer-targeted therapy with low-level ultrasound, SDT, has been introduced. The collapse of cavities can produce interesting chemical effects, known as sonochemistry. In order to develop effective SDT, evaluation of effective parameters is the necessary concluding type of ultrasound mode and intensity. 


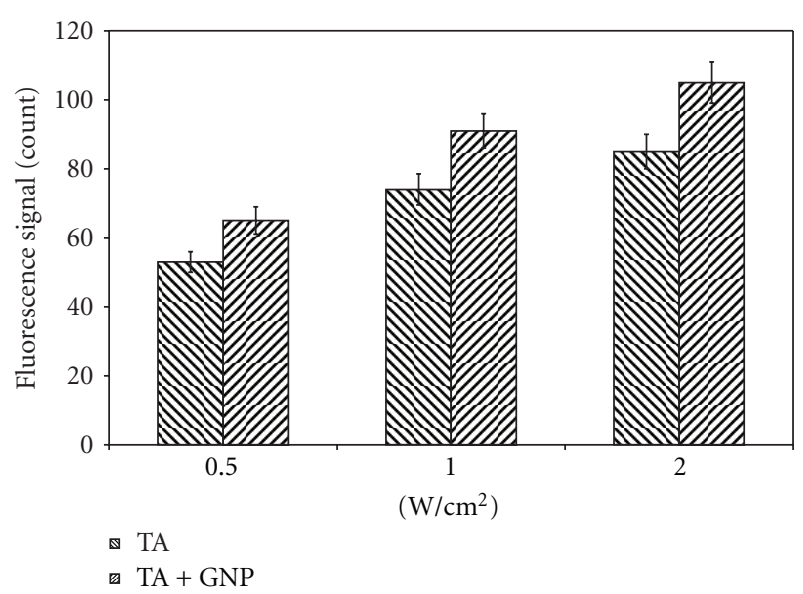

FIGURE 3: Recorded fluorescence signal intensity in the TA solutions in the absence and presence of GNPs at $0.5,1$, and $2 \mathrm{~W} / \mathrm{cm}^{2}$ intensities in continuous mode, excitation wavelength $=310 \mathrm{~nm}$, and emission and excitation band width $=5 \mathrm{~nm}$.

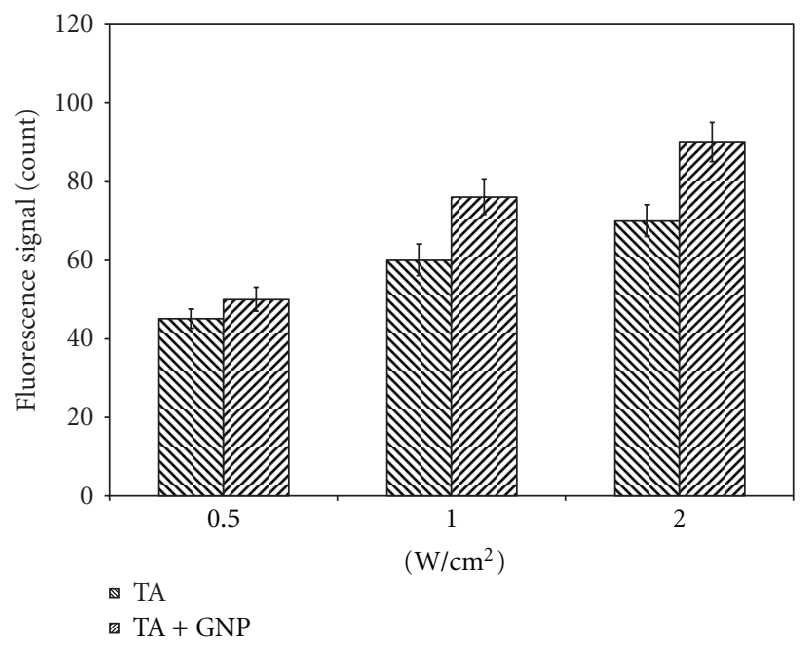

FIGURE 4: Recorded fluorescence signal intensity in the TA solutions in the absence and presence of GNPs at $0.5,1$, and $2 \mathrm{~W} / \mathrm{cm}^{2}$ intensities in pulsed mode (in duty cycle of 50\%), excitation wavelength $=310 \mathrm{~nm}$, and emission and excitation band width $=$ $5 \mathrm{~nm}$.

In this study hydroxyl radical production was measured in the field of $1 \mathrm{MHz}$ ultrasound waves at low-level intensity by the TA dosimetry method. This method of dosimetry is specific for trapping of hydroxyl radical production in fields of ultrasound irradiation, because each molecule of hydroxyl radical is trapped by one molecule of TA and forms one molecule of HTA which can be measured by the fluorescence spectroscopy method. Because this method of dosimetry is based on the fluorimetry method, it is very sensitive for hydroxyl radical measurement.

Barati et al. showed that the TA dosimetry is suitable for detecting and quantifying free hydroxyl radicals as a criterion of cavitation production in medical ultrasound fields [12].

In the present study, we have investigated the cavitation potential of the GNPs by this method.

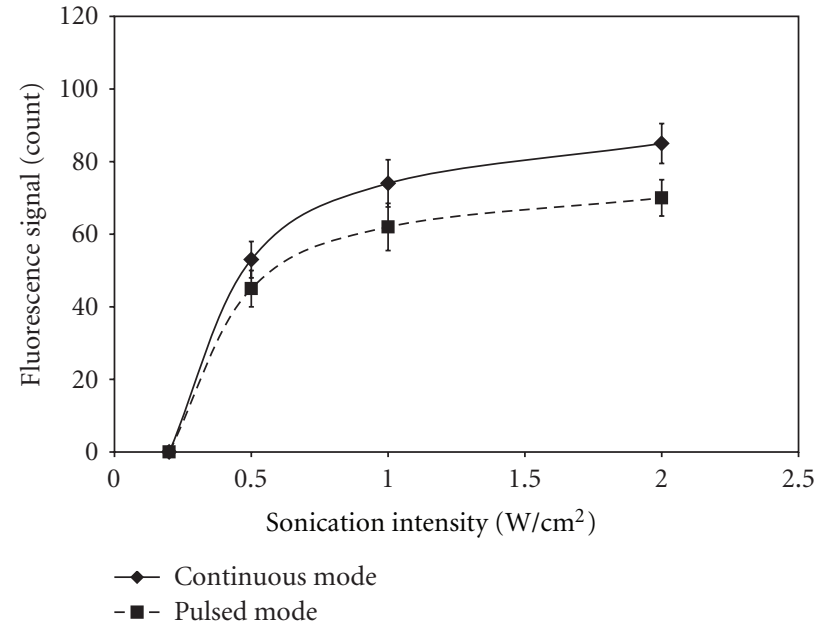

FIGURE 5: Recorded fluorescence signal from the TA solution without gold nanoparticles in the field of $1 \mathrm{MHz}$ ultrasound waves at intensities of $0.5,1$, and $2 \mathrm{~W} / \mathrm{cm}^{2}$ in continuous and pulsed (in duty cycle of $50 \%$ ) modes, excitation wavelength $=310 \mathrm{~nm}$, and emission and excitation band width $=5 \mathrm{~nm}$.

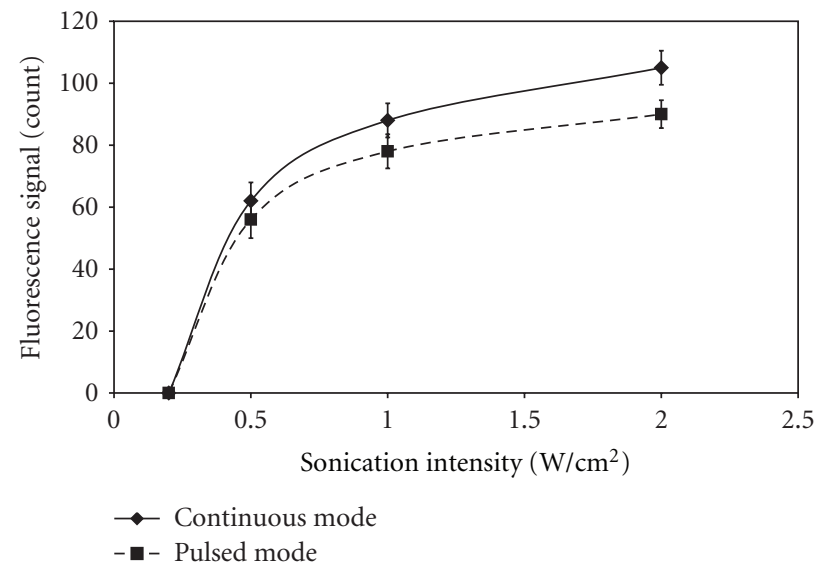

FIGURE 6: Recorded fluorescence signal from the TA solution containing gold nanoparticles in the field of $1 \mathrm{MHz}$ ultrasound waves at intensities of $0.5,1$, and $2 \mathrm{~W} / \mathrm{cm}^{2}$ in continuous and pulsed (in duty cycle of $50 \%$ ) modes, excitation wavelength $=310 \mathrm{~nm}$, and emission and excitation band width $=5 \mathrm{~nm}$.

Our results showed that the fluorescence signal intensity for TA solution containing GNPs was higher than the TA solution without GNPs in the different intensities in continuous mode. It should be noted that the above phenomenon is amplified by continuous ultrasound waves with the intensity of $2 \mathrm{~W} / \mathrm{cm}^{2}$.

This finding could have been related to two courses of action: (1) GNPs acted as cavitation nuclei, that is, the nanoparticles may have acted as the sites for cavitation and increased the cavitation rate [6]; (2) increased collapse of cavities could have been another feasible process.

Consequently, many more $\mathrm{OH}$ radicals are created resulting in the higher level of the fluorescence emission. 
Therefore, it is predicted that GNPs can increase the cavitation rate in the TA solution containing GNPs.

Tuziuti et al. showed that the existence of particles in a liquid provides a nucleation site for the cavitation bubble due to its surface roughness, and it leads to decrease in the cavitation threshold responsible for the increase in the quantity of bubbles, when the liquid is irradiated by ultrasound [6].

Since SDT is performed in temperatures below the hyperthermia effect threshold on biological environments therefore, in all experiments, temperature changes must be less than $2-3^{\circ} \mathrm{C}$.

The results of experiments related to the sonication mode for $1 \mathrm{MHz}$ ultrasound irradiation in the different intensities show that the fluorescence signal in continuous mode of sonication is $20 \%$ higher than the pulsed mode in a $50 \%$ duty cycle for SDT. This is due to the dosage of energy released by ultrasound irradiation in continuous mode.

The amount of hydroxyl radicals production versus ultrasound intensity show that, with increasing intensity in continuous and pulsed modes, the hydroxyl radical production is increased.

Experimental results obtained by sonication of the TA solutions have indicated that the ultrasound irradiation parameters such as mode of exposure, intensity, and nucleation sites for bubbles generation are effective in hydroxyl radical production and, in turn, in the production of cavitation.

It should be noted that the above phenomenon is amplified by continuous ultrasound waves with the intensity of $2 \mathrm{~W} / \mathrm{cm}^{2}$.

\section{Conclusion}

Acoustic cavitation in the presence of GNPs has been introduced as a way for improving therapeutic effects on the tumors in SDT. TA dosimetry is a suitable method for monitoring the acoustic cavitation effects by measurement of hydroxyl radicals in medical ultrasound ranges. It should be remembered, however, that this method employs a chemical dosimeter and as such it may be the only ideal dosimeter for estimation of cavitation production.

\section{References}

[1] T. Yu, Z. Wang, and T. J. Mason, "A review of research into the uses of low level ultrasound in cancer therapy," Ultrasonics Sonochemistry, vol. 11, no. 2, pp. 95-103, 2004.

[2] I. Rosenthal, J. Z. Sostaric, and P. Riesz, "Sonodynamic therapya review of the synergistic effects of drugs and ultrasound," Ultrasonics Sonochemistry, vol. 11, no. 6, pp. 349-363, 2004.

[3] N. Yumita, R. Nishigaki, and S. I. Umemura, "Sonodynamically induced antitumor effect of Photofrin II on colon 26 carcinoma," Journal of Cancer Research and Clinical Oncology, vol. 126, no. 10, pp. 601-606, 2000.

[4] H. Tang, C. C. J. Wang, D. Blankschtein, and R. Langer, "An investigation of the role of cavitation in low-frequency ultrasound-mediated transdermal drug transport," Pharmaceutical Research, vol. 19, no. 8, pp. 1160-1169, 2002.
[5] P. Marmottant and S. Hilgenfeldt, "Controlled vesicle deformation and lysis by single oscillating bubbles," Nature, vol. 423, no. 6936, pp. 153-156, 2003.

[6] T. Tuziuti, K. Yasui, M. Sivakumar, Y. Iida, and N. Miyoshi, "Correlation between acoustic cavitation noise and yield enhancement of sonochemical reaction by particle addition," Journal of Physical Chemistry A, vol. 109, no. 21, pp. 48694872, 2005.

[7] C. H. Farny, T. Wu, R. G. Holt, T. W. Murray, and R. A. Roy, "Nucleating cavitation from laser-illuminated nano-particles," Acoustic Research Letters Online, vol. 6, no. 3, pp. 138-143, 2005.

[8] X. Huang, P. K. Jain, I. H. El-Sayed, and M. A. ElSayed, "Plasmonic photothermal therapy (PPTT) using gold nanoparticles," Lasers in Medical Science, vol. 23, no. 3, pp. 217-228, 2008.

[9] G. F. Paciotti, L. Myer, D. Weinreich et al., "Colloidal gold: a novel nanoparticle vector for tumor directed drug delivery," Drug Delivery, vol. 11, no. 3, pp. 169-183, 2004.

[10] J. C. Y. Kah, M. C. Olivo, C. G. L. Lee, and C. J. R. Sheppard, "Molecular contrast of EGFR expression using gold nanoparticles as a reflectance-based imaging probe," Molecular and Cellular Probes, vol. 22, no. 1, pp. 14-23, 2008.

[11] H. Hasanzadeh, M. Mokhtari-Dizaji, S. Z. Bathaie, and Z. M. Hassan, "Evaluation of correlation between chemical dosimetry and subharmonic spectrum analysis to examine the acoustic cavitation," Ultrasonics Sonochemistry, vol. 17, no. 5, pp. 863-869, 2010.

[12] A. H. Barati, M. Mokhtari-Dizaji, H. Mozdarani, S. Z. Bathaei, and Z. M. Hassan, "Free hydroxyl radical dosimetry by using $1 \mathrm{MHz}$ low level ultrasound waves," Iranian Journal of Radiation Research, vol. 3, no. 4, pp. 163-169, 2006.

[13] C. Haosheng, W. Jiadao, and C. Darong, "Cavitation damages on solid surfaces in suspensions containing spherical and irregular microparticles," Wear, vol. 266, no. 1-2, pp. 345-348, 2009.

[14] R. Bhattacharya, P. Mukherjee, Z. Xiong, A. Atala, S. Soker, and D. Mukhopadhyay, "Gold nanoparticles inhibit VEGF165-induced proliferation of HUVEC cells," Nano Letters, vol. 4, no. 12, pp. 2479-2481, 2004.

[15] G. J. Price, F. A. Duck, M. Digby, W. Holland, and T. Berryman, "Measurement of radical production as a result of cavitation in medical ultrasound fields," Ultrasonics Sonochemistry, vol. 4, no. 2, pp. 165-171, 1997.

[16] T. J. Mason, J. P. Lorimer, D. M. Bates, and Y. Zhao, "Dosimetry in sonochemistry: the use of aqueous terephthalate ion as a fluorescence monitor," Ultrasonics Sonochemistry, vol. 1, no. 2, pp. S91-S95, 1994. 


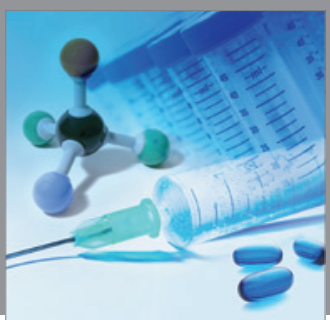

International Journal of

Medicinal Chemistry

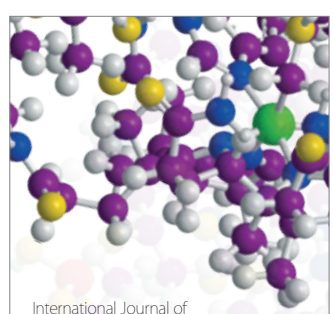

Carbohydrate Chemistry

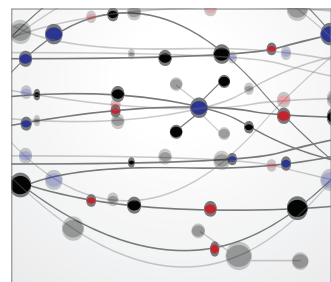

The Scientific World Journal
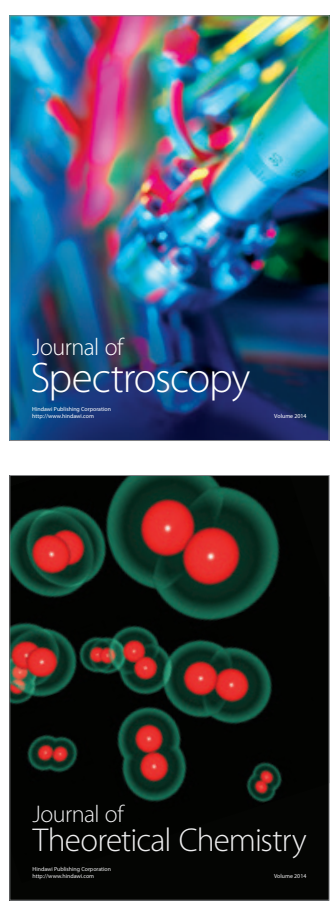
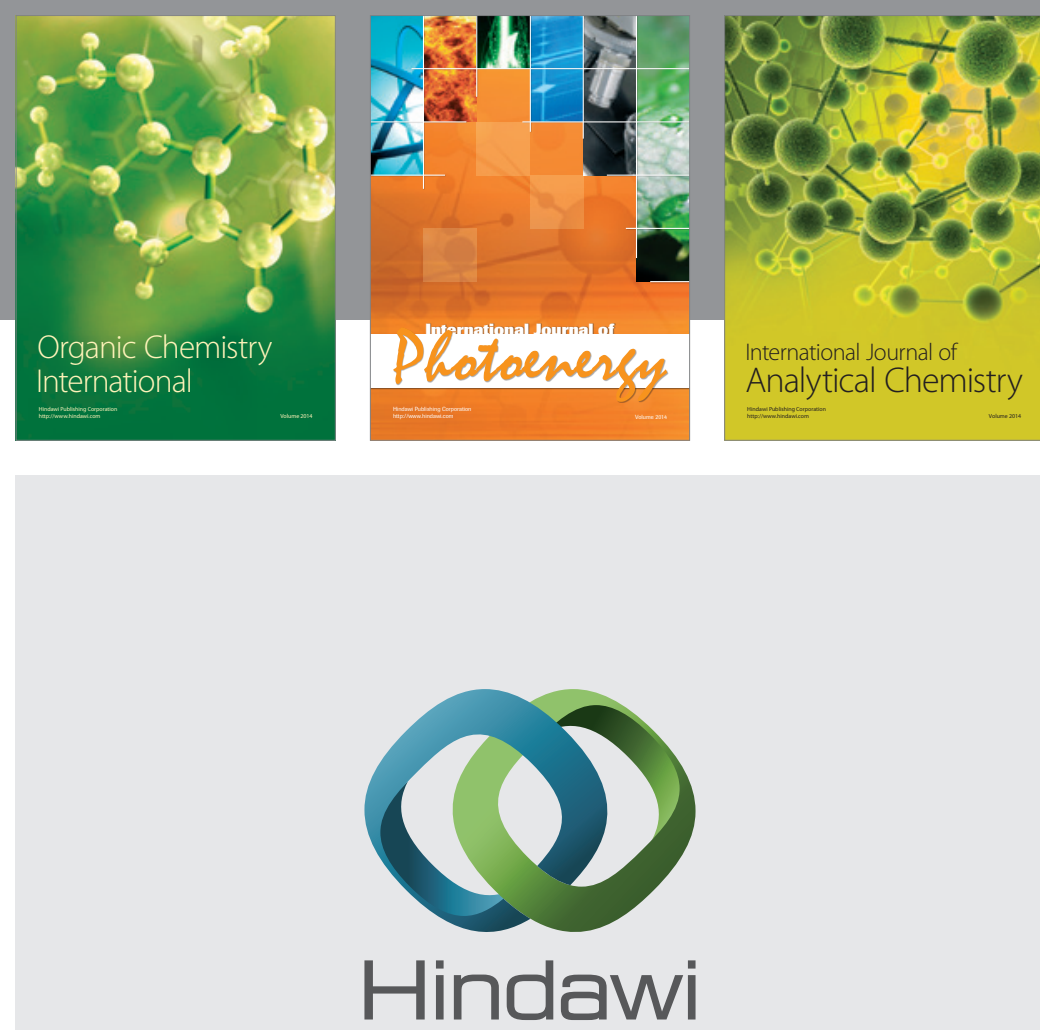

Submit your manuscripts at

http://www.hindawi.com
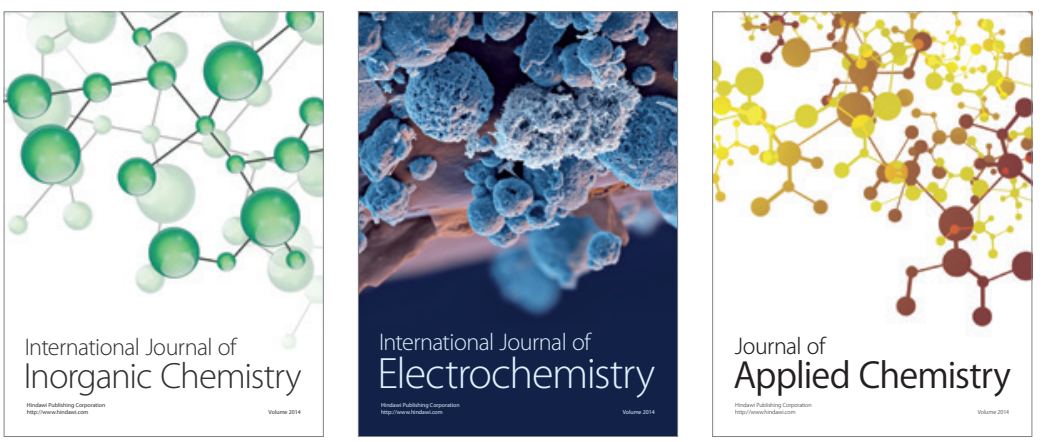

Journal of

Applied Chemistry
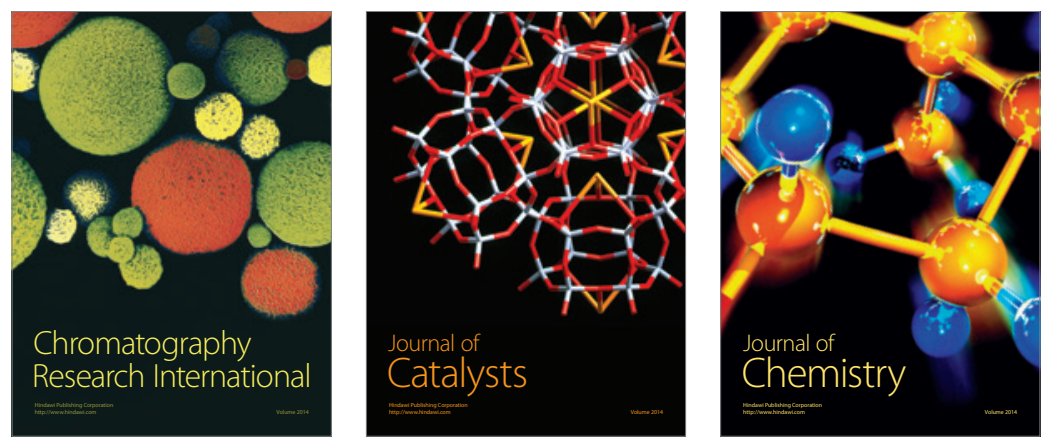
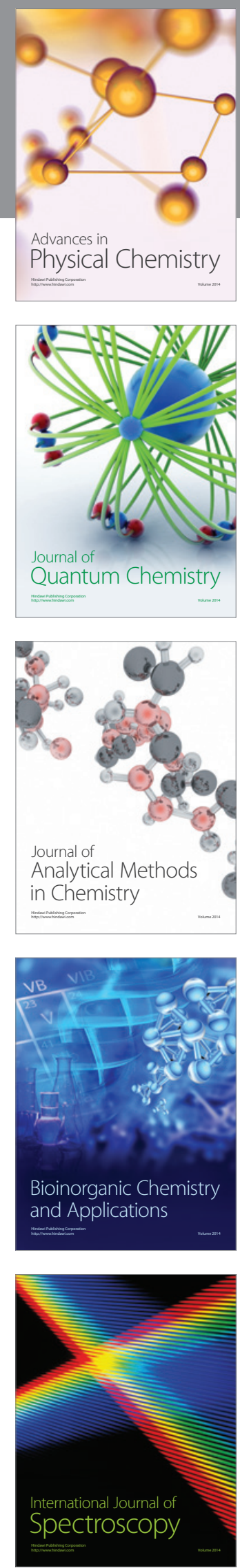